\title{
Laser heating of a dense plasma pinch
}

\author{
David L. Chapin* and James J. Duderstadt \\ Department of Nuclear Engineering, The University of Michigan, Ann Arbor, Michigan 48105 \\ (Received 10 May 1974; final manuscript received 11 November 1974)

\begin{abstract}
A single fluid, two-temperature, one-dimensional magnetohydrodynamic model has been developed to describe the properties of a linear pinch plasma heated by an axially directed laser beam. The coupling of the laser light energy with the pinch dynamics is studied in detail. The laser light absorption occurs primarily through the inverse bremsstrahlung process, but the effect of enhanced absorption for sufficiently intense lasers has also been studied. Results of the calculations show a strong interaction between the heating by radial compression (pinching) and heating by the laser beam. It was found that the most important considerations in obtaining efficient heating of the plasma are the time profiles of the laser pulse and the electric current. The results of the anomalous absorption studies indicate that the increased absorption can cause the electron and ion temperatures to decouple and may actually be detrimental to the efficient laser heating of dense pinch type plasmas.
\end{abstract}

\section{INTRODUCTION}

A very interesting alternative to the laser-driven compression ${ }^{1}$ of fuel pellets in laser fusion involves the use of long wavelength lasers to heat lower density plasmas which can be confined in pulsed magnetic fields. ${ }^{2,3}$ While the use of laser heating does not eliminate the usual plasma confinement problem (as it does in pellet compression), it does provide an alternative method of rapidly heating plasmas to thermonuclear temperatures. This allows one to work with higher plasma densities and volumes than achievable with more conventional $\theta$ or $z$ pinches. Furthermore, high powered $\mathrm{CO}_{2}$ lasers (with wavelengths of $10.6 \mu$ ) presently exist ${ }^{4}$ with the proper pulse lengths $(10-100 \mu \mathrm{sec})$ and pulse energies $\left(10^{3}-\right.$ $\left.10^{4} \mathrm{~J}\right)$ to provide significant heating of dense pinches $\left(10^{17}\right.$ to $10^{18}$ ions $\left./ \mathrm{cm}^{3}\right)$. Also, there appears to be no fundamental difficulty in building these long pulse $\mathrm{CO}_{2}$ lasers with considerably higher energies $(>10 \mathrm{~kJ}) .^{5}$

There have been some preliminary experiments performed using low powered $\mathrm{CO}_{2}$ lasers to heat magnetically confined plasmas. ${ }^{6,7}$ When similar experiments are performed in the future with higher energy lasers, they may demonstrate that laser heating of pinches is a practical method of achieving fusion temperatures. Previous theoretical studies ${ }^{8,9}$ of this plasma heating scheme have usually been based on overly simplified models that often neglect important physical phenomena. As such, these studies were intended to give only approximate ideas of the practicality of using long pulse $\mathrm{CO}_{2}$ lasers to heat dense pinches.

In this paper we present the results of a detailed, theoretical analysis of the laser heating of dense pinch plasmas. We utilize a one-dimensional, single fluid, two-temperature magnetohydrodynamic model of the plasma. The resulting equations are solved numerically by a computer code. A source term to account for heating by an axially directed laser beam is added to the electron energy equation. This term assumes the laser light absorption is via inverse bremsstrahlung, although the effect of an increase in the absorption resulting from possible anomalous absorption mechanisms has also been studied. Variations in the temporal dependence of the laser pulse shape have also been investigated.
Our calculations are made for relatively short laser pulses $(\sim 1 \mu \mathrm{sec})$, while a reactor would require confinement times on the order of $10^{-3}-10^{-4} \mathrm{sec}$. Hence, our analysis applies only to the early stages of the heating, when the laser light absorption would be most important.

\section{MAGNETOHYDRODYNAMIC MODEL}

The fluid model is based on the method of describing the plasma as a hydromagnetic fluid, with separate electron and ion temperatures and both finite thermal conductivity and electrical resistivity. The equations comprising the model are $\mathrm{e}^{10,11}$

$$
\begin{aligned}
\frac{D v}{D t}= & v \nabla \cdot \mathbf{u}, \\
\frac{D \mathbf{u}}{D t}= & -v \nabla\left(p_{i}+p_{e}\right)+v \mathbf{J} \times \mathbf{B}, \\
\frac{D e_{e}}{D t}= & -p_{e} v \nabla \cdot \mathbf{u}+v \nabla \cdot\left(\kappa_{e} \nabla T_{e}\right)+Q_{L} \\
& +v \mathbf{J} \cdot(\eta \mathbf{J})-\frac{\partial e_{e}}{\partial T_{e}} \frac{\left(T_{e}-T_{i}\right)}{t_{\mathrm{eq}}}, \\
\frac{D e_{i}}{D t}= & -p_{i} v \nabla \cdot \mathbf{u}+v \nabla \cdot\left(\kappa_{i} \nabla T_{i}\right)+\frac{\partial e_{i}}{\partial T_{i}} \frac{\left(T_{e}-T_{i}\right)}{t_{\text {eq }}}, \\
\frac{\partial \mathbf{B}}{\partial t}= & -\nabla \times\left(\frac{\eta}{4 \pi} \nabla \times \mathbf{B}\right)+\nabla \times(\mathbf{u} \times \mathbf{B}),
\end{aligned}
$$

and

$$
p_{\alpha} v=R T_{\alpha} ; e_{\alpha}=\frac{3}{2} R T_{\alpha}(\alpha=e, i),
$$

where

$$
\frac{D f(\mathbf{r}, t)}{D t} \equiv \frac{\partial f}{\partial t}+(\mathbf{u} \cdot \nabla) f .
$$

Here and elsewhere, $\eta$ is the resistivity, $B$ is the magnetic field, $\mathbf{u}$ is the velocity, $v$ is the specific volume, $R$ is the gas constant, $t_{\text {eq }}$ is electron-ion equilibration time, and $p_{\alpha}, e_{\alpha}, \kappa_{\alpha}$, and $T_{\alpha}$ are, respectively, the pressure, internal energy, thermal conductivity, and temperature of the electrons $(\alpha=e)$ and ions $(\alpha=i)$. In the electron energy equation the $Q_{L}$ term is added to account for heating by the laser $\left(Q_{L}\right.$ is discussed in Sec III.). We assume a fully ionized plasma with charge 
neutrality and no charge separation.

The equations are then expanded in one-dimensional, cylindrical geometry, with symmetry assumed in the $\Theta$ and $z$ directions. Hence, neglecting end effects, only radial variations and time dependence are allowed. Since the model is one dimensional and does not allow for the axial transfer of heat along the plasma column, the length of the pinches that are simulated is small so that the laser light is uniformly absorbed along the length of the plasma. We choose to describe the plasma in the Lagrangian frame of reference. We have written a computer code to solve the resulting set of coupled partial differential equations. Two separate codes were developed to study $\Theta$ and $z$ pinch plasmas. The diffusion type equations for $B, T_{e}$, and $T_{i}$ were solved with an implicit numerical scheme, while an explicit solution was used to solve for $u$ and $v$. The forms for the equilibration time $t_{\mathrm{eq}}$ and resistivity $\eta$ are taken from Spitzer, ${ }^{12}$ while the forms for the electron and ion thermal conductivities $\kappa_{\alpha}$ are taken from Braginskii. ${ }^{13}$ To prevent discontinutiies in the region of shocks during the numerical computation, the usual artificial viscosity ter $\mathrm{m}^{14}$ is added to the ion temperature equation and the momentum equation.

\section{LASER SOURCE TERM}

The laser energy deposition rate per gram $Q_{L}$ to a plasma of length $L$ is given by

$$
Q_{L}=\frac{v I_{0}}{L}\left(1-e^{-\kappa L}\right) \mathrm{W} / \mathrm{g},
$$

where $I_{0}$ is the laser beam intensity $\left(\mathrm{W} / \mathrm{cm}^{2}\right)$ and $\kappa\left(\mathrm{cm}^{-1}\right)$ is the absorption coefficient. For the numerical calculation of $Q_{L}$, special care is taken to determine which zones of the plasma mesh are covered by the focal area of the laser beam. In using Eq. (8) for $Q_{L}$, we have assumed that the beam is uniformly absorbed along the plasma column. For this to be true, we require $\kappa^{-1}$ $>L$. As a result of this requirement, the length of the pinches that can be simulated by this laser heating model is generally fairly small $(\sim 50 \mathrm{~cm})$. Thus, the analysis pertains more to the results of a small scale experiment than a possible reactor configuration where lengths of several kilometers may be necessary. ${ }^{2,3}$ To analyze such a long device, the axial transfer of heat energy and attenuation of the incident laser beam would certainly have to be included. However, our analysis could be regarded as applying to a small section of a very long reactor. Possible refraction and reflection effects ${ }^{15}$ due to trapping of the laser beam in a long plasma column have not been included in our absorption model.

For most parts of the analysis we assumed that inverse bremsstrahlung was the process by which the laser radiation was absorbed by the plasma electrons. The classical value for the absorption coefficient $k$ characterizing this collisional absorption mechanism is $^{12}$

$$
\kappa=4.4 \times 10^{-38} \frac{n_{1}^{2} \lambda_{0}^{2} \ln \Lambda}{T_{e}^{3 / 2}} \frac{1}{N_{i}} \mathrm{~cm}^{-1},
$$

where $n_{i}$ is the density $\left(\mathrm{cm}^{-3}\right), \lambda_{0}$ is the laser wavelength $(\mu), \ln \Lambda$ is the standard Coulomb logarithm term, ${ }^{12}$
$T_{e}$ is the electron temperature $(\mathrm{eV})$, and $N_{i}$ is the index of refraction. In the calculation a check is performed to determine whether the plasma density is less than the critical density. If it is not, then the laser energy addition term $Q_{L}$ is set to zero, and we assume the incident beam is simply reflected back from the plasma.

If the incident laser beam is of sufficiently high intensity, the interaction of the radiation with the plasma may be altered by nonlinear processes. ${ }^{16}$ The classical $k$ can be altered by these anomalous effects, possibly resulting in an increase in the heating rate. For most of the pinches analyzed here, the plasma was underdense, that is, $\omega_{0} \gg \omega_{p e}$, where $\omega_{\text {pe }}$ is the electron plasma frequency and $\omega_{0}$ is the laser frequency. This rules out many of the well-known parametric instability processes $^{17}$ which occur only in the vicinity of $\omega_{0} \simeq \omega_{\text {pe }}$. However, there are other collective instability mechanisms that can occur in underdense plasmas, such as the electron wave or $2 \omega_{p e}$ instability. ${ }^{18}$ Other types of instabilities with $\omega_{0} \% \omega_{p e}$ have been investigated by $\operatorname{Silin}^{19}$ and Kruer. ${ }^{20}$ Stimulated scattering processes ${ }^{21}$ may also occur in such underdense plasmas. Hence, there are a number of possible instability mechanisms that may result in anomalous heating of underdense plasmas. In our calculations, we developed two different approaches to simulate the effects of possible anomalous heating. In the first method, the classical $\kappa$ given in Eq. (9) is simply scaled by multiplying it by a constant. For the second method, we defined a cutoff temperature $T_{c}$ such that when $T_{e}>T_{c}$ the absorption coefficient does not depend on temperature. This technique is related to the procedure of replacing the collision frequency in $\kappa$ by the ion plasma frequency. ${ }^{22}$ When these anomalous absorption models were used, the transport coefficients such as resistivity and thermal conductivity were kept at their classical values.

It was also of interest to study the variations in pinch heating as the temporal shape of the laser pulse was varied. To investigate different pulse shapes, we kept the total laser pulse energy a constant and studied how the energy was absorbed by the plasma for various laser pulse shapes. In all calculations the spatial profile of the beam was assumed to be constant across the focal area.

\section{LASER HEATED $z$ PINCH}

For the first part of the investigation, we used the fluid model to study the $\mathrm{CO}_{2}$ laser heating of a $z$ pinch to temperatures approaching those necessary for fusion. The plasma in the pinch was assumed to be deuterium with an initial density of $5 \times 10^{17} \mathrm{~cm}^{-3}$ and initial diameter of $0.8 \mathrm{~cm}$. The initial temperature of both the electrons and ions was taken to be $10 \mathrm{eV}$. The electrical current flowing in the axial direction was a step function of magnitude $3.92 \times 10^{5} \mathrm{~A}$ for $t<0.3 \mu \mathrm{sec}$, and then jumped to $5.8 \times 10^{5} \mathrm{~A}$ for $t>0.3 \mu \mathrm{sec}$. The current was chosen of this form so that when the plasma began to expand appreciably during the early heating phase, the current would increase and the resulting increase in the magnetic field would be able to contain the plasma as it was heated still further. 


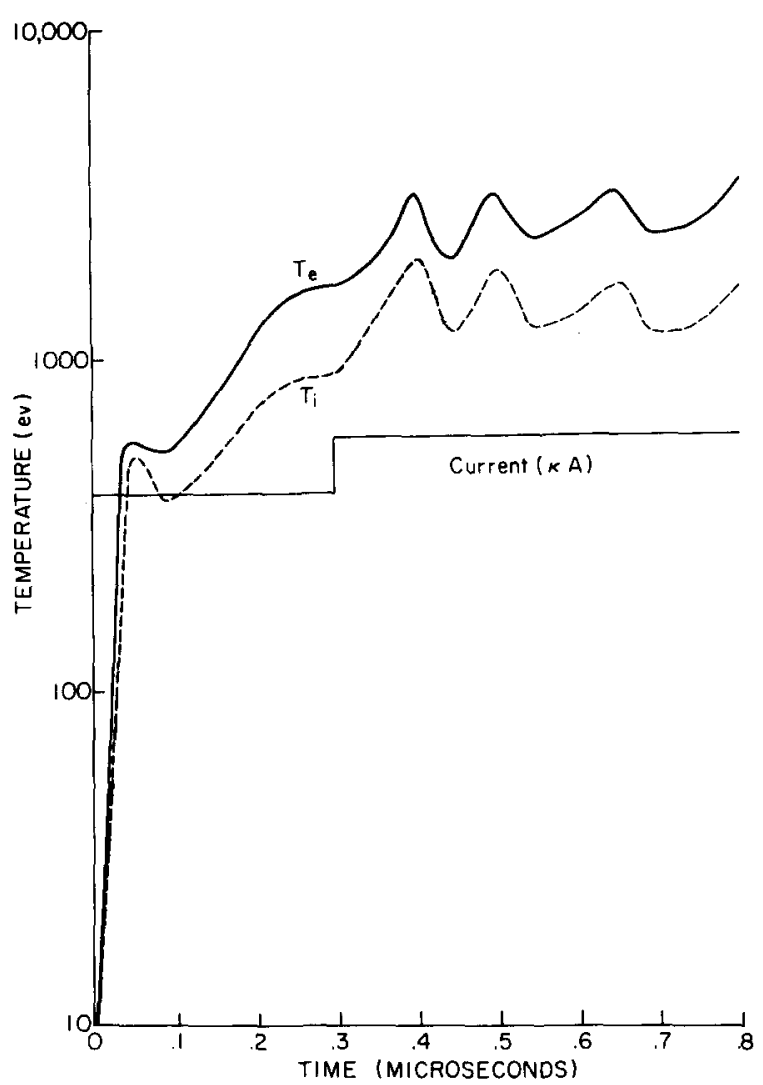

FIG. 1. Laser heated $z$ pinch temperatures.

The $\mathrm{CO}_{2}$ laser light directed along the plasma axis had a constant power of $5 \times 10^{11} \mathrm{~W}$ for the duration of the analysis $(0.8 \mu \mathrm{sec})$, corresponding to a $400 \mathrm{~kJ}$ laser. It was focused to a spot of $0.5 \mathrm{~mm}$, resulting in a constant incident laser intensity of $6.4 \times 10^{13} \mathrm{~W} / \mathrm{cm}^{2}$ 。 The length of the pinch was $50 \mathrm{~cm}$. In Fig. 1 the average electron and ion temperatures predicted by the code are plotted vs time. The electrical current is also shown. The plasma heats very quickly at first, with the temperature rising to over $500 \mathrm{eV}$ in the first $50 \mathrm{nsec}$. The plasma then expands slightly and the temperature drops before it again begins to rise smoothly to over $1 \mathrm{keV}$. At the time of the current jump $(0.3 \mu \mathrm{sec})$, the temperature again rises, reaching electron temperatures of over $3 \mathrm{keV}$ and ion temperatures of nearly $2 \mathrm{keV}$. During the later stages of the run the temperatures are found to oscillate as the plasma undergoes slight pinches and expansions, with the electrons being about $1 \mathrm{keV}$ higher than the ions. The reason for the fast rise in the temperatures is that the laser energy is deposited in the plasma very quickly, with over $75 \%$ of the total laser energy addition being deposited within $400 \mathrm{nsec}$. From about $400 \mathrm{nsec}$ to the termination of the run ( $800 \mathrm{nsec})$, the plasma was found to have reached a nearly steadystate condition, with peaks in the temperatures occurring only when the plasma is pinched. This indicates that during this time range the compression heating was most important, with the laser heating being relatively ineffective due to the high temperatures. This behavior arises because of the decrease in $K$ as $T_{e}$ increases.

The laser energy chosen for these studies was $400 \mathrm{~kJ}$ distributed over a pulse length of $0.8 \mu \mathrm{sec}$. However, since the plasma was found to have reached a near steadystate by $0.8 \mu \mathrm{sec}$ with little energy being absorbed from the laser at that time, it might be possible to stop the laser earlier (perhaps $0.4 \mu \mathrm{sec}$ ) and simply confine the hot plasma with the current at later times. The average ion density was about $10^{18} \mathrm{~cm}^{-3}$, which means that the plasma would have to be confined for about $100 \mu \mathrm{sec}$ to reach the Lawson criterion. If the current could shock heat the plasma to slightly higher temperatures during this confinement time, it would be possible to reach fusion conditions even though the laser was turned off early. Also, if fusion were initiated, the plasma could be self-heated by the alpha particles to produce more efficient fusion burn. Thus, in this scheme the laser would act only as an ignition device to heat the plasma at the early times before it becomes sufficiently hot such that little additional laser light absorption is possible. Hence, the requirements on the laser would not be excessive since it is needed only for a relatively short time. Due to economic limitations with the run time of the code, only the first step in such a process, the laser heating, was investigated in this analysis.

In the heating of the $z$ pinch shown in Fig. 1, the laser beam was focused on a very small area with a focal radius $r_{L}$ of $0.5 \mathrm{~mm}$. This small area is less than $0.5 \%$ of the initial plasma area with diameter $0.8 \mathrm{~cm}$. Since a fairly uniform distribution of temperature across the plasma was desired, it was necessary to study the results of heating only a small cross sectional area of the plasma with the laser. The radial distributions of the electron and ion temperatures at three different times are shown in Fig. 2. From the plot it is clear that the

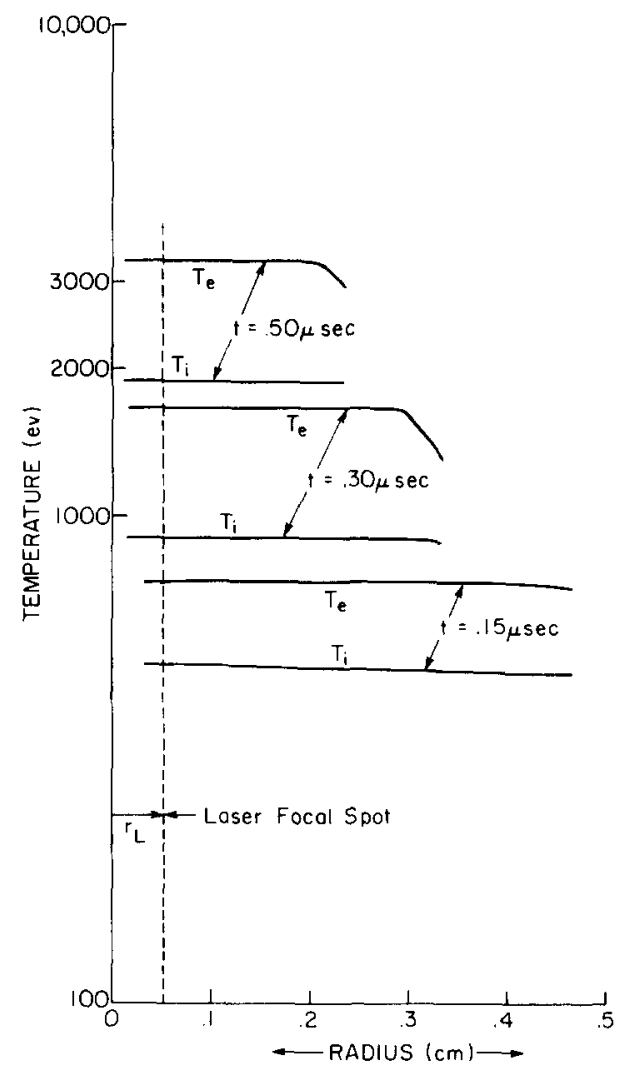

FIG. 2. Laser heated $z$ pinch temperature profiles. 


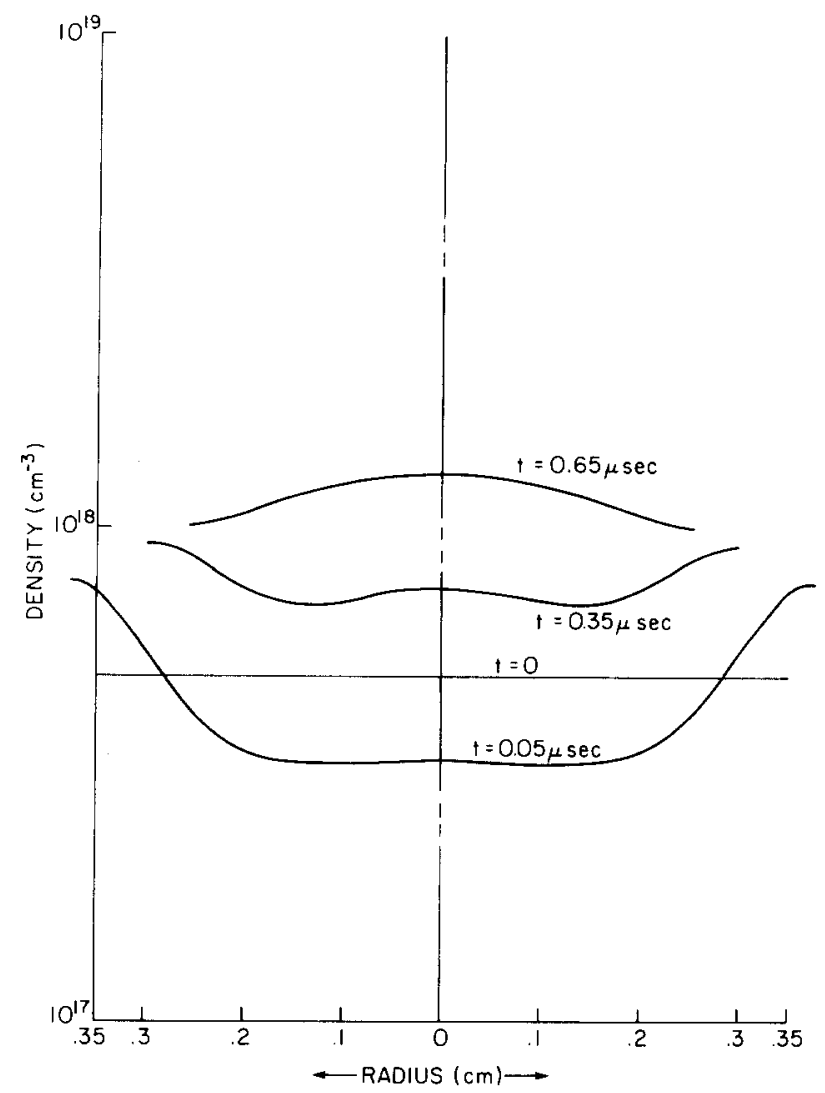

FIG. 3. Laser heated $z$ pinch density profiles.

temperatures are nearly constant across the plasma, with the slight exceptions of $T_{\mathfrak{e}}$ near the boundary at the two later times. Thus, the energy deposited at the center is quickly spread across the plasma. The physical process responsible for this is electron thermal conduction. It was found that the thermal conduction times (i.e., the time necessary for a thermal diffusion wave to travel across the plasma) were on the order of nanoseconds or less, and hence this process was rapid enough to distribute the energy evenly. The hot electrons then transferred some of their energy to the ions through the collision term involving $t_{\mathrm{eq}}$ in the temperature equations, and thus the ions were also found to have smooth temperature profiles.

Another quantity of interest in the plasma is the radial profile of the plasma density, since it has been shown ${ }^{15}$ that the shape of the profile can affect the refraction of the laser beam and possibly reduce the effective collision length of the laser beam. In Fig. 3 the calculated radial density profile is shown for three different times, along with the initial profile. At early times, when the laser absorption was the strongest, the profile was found to be favorable, ${ }^{15}$ that is, with a minimum on or near the axis. However, at later times the plasma often had an unfavorable profile with a maximum on the axis. The formation of these density profiles results from the pinching and expansion of the plasma column, with a pinch causing an increase in the density on the outer part of the plasma to form the favorable profile.

It was also of interest to compare the behavior of this $z$-pinch plasma with the situation when no laser heating was used. To study this, the same initial and boundary conditions on the plasma were used, but the laser source was removed. The prediction of this plasma showed a very interesting behavior. It was found ${ }^{23}$ that within 10 nsec the ion temperature rose to nearly $2 \mathrm{keV}$ before it quickly dropped of to less than $100 \mathrm{eV}$. The reason for the large rise in $T_{i}$ is a very strong shock compression at the beginning that results from the high electrical current. This leads to an increase in the plasma pressure which drives the plasma outward in an expansion that cools it to low temperatures. The same process was evident with the electrons, although $T_{e}$ is less than $T_{i}$ because the shock energy is deposited in the ions. Thus, it is clear that the laser serves a second purpose besides heating the plasma; that is, it can prevent a quick compression of the plasma by adding energy to it and raising the plasma particle pressure. This creates a balance between the particle and field pressures and allows a more uniform heating of the plasmaby the laser.

We have investigated the possible effects of anomalous laser heating of the $z$ pinch by scaling the absorption coefficient by 10 and 50 times its classical value. It was found ${ }^{23}$ that $T_{e}$ rises very quickly, as one might expect. However, this sudden rise is off set by a plasma expansion that follows, so that by about $250 \mathrm{nsec}$ the electron temperature was nearly the same as the classical case. The ion temperature was found to be much lower for the increased absorption cases, due to the sudden rise in $T_{e}$ which causes the equilibration time to reach a large value very quickly. Since $t_{e q}$ is suddenly very large, there is very little transfer of energy from the hot electrons to the ions, and hence the ions remain relatively cold. These results tend to show that anomalous heating processes may be undesirable in attempting to laser heat a magnetically confined plasma. This is particularly true if the increased heating occurs too rapidly, in which case the electrons and ions may decouple due to the increased equilibration time.

\section{LASER HEATED $\theta$ PINCH}

As a second part of the investigation we used the fluid code to study $\mathrm{CO}_{2}$ laser heating of $\theta$ pinches. We first investigated the effect of changing the laser pulse shape. A deuterium plasma was again assumed with an initial density of $5 \times 10^{17} \mathrm{~cm}^{-3}$, initial diameter of $5.4 \mathrm{~cm}$, and initial electron and ion temperatures of $2 \mathrm{eV}$. The plasma length was $20 \mathrm{~cm}$, and the electric current was a sine function with a peak value of $3.5 \times 10^{6} \mathrm{~A}$ occurring at $2.0 \mu \mathrm{sec}$. The $\mathrm{CO}_{2}$ laser directed along the axis was focused to a spot of $0.5 \mathrm{~mm}$ radius. Four different laser pulse profiles varying as $t^{m}$ were used: constant $(\mathrm{m}=0)$, linear $(\mathrm{m}=1)$, quadratic $(\mathrm{m}=2)$, and cubic $(m=3)$. The peak powers were varied for each shape so that the total laser energy delivered to the plasma for each case was $2.5 \mathrm{~kJ}$ for a duration of $1 \mu \mathrm{sec}$, resulting in intensities on the order of $10^{12} \mathrm{~W} / \mathrm{cm}^{2}$. As shown in Fig. 4, the electron temperature was higher for lower values of $m$, while $T_{i}$ was highest for higher values of $m$. The reason for this difference in $T_{e}$ and $T_{i}$ is the temporal behavior of the laser pulse. For example, for the $m=0$ case a relatively large amount of laser energy 


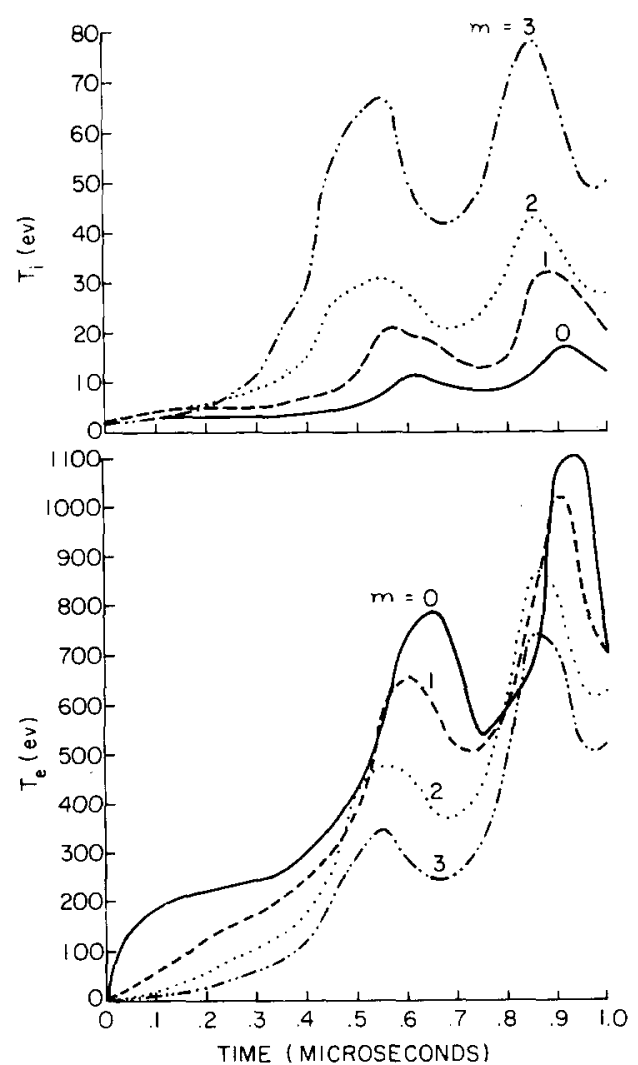

FIG. 4. Theta pinch electron and ion temperatures for different laser pulse shapes.

is deposited in the electrons at the beginning. This large and fast heating of the electrons results in the equilibration time $t_{\text {e }}$ becoming large very quickly. The increase of $t_{\text {eq }}$ causes only a small portion of the electron energy to be transferred to the ions. Also, since the current was a sine function, it was small at the start and was not able to prevent the plasma from expanding due to the laser energy addition. Hence, a large part of the absorbed energy went into directed motion of expansion with little being converted into internal energy of the plasma. The opposite behavior was found for the cubic $(m=3)$ pulse, where the laser power rose slowly. Here, the electron temperature was low at the beginning and the slow deposition of energy by the laser prevented the plasma column from expanding. The pinching and slow rise of $T_{e}$ resulted in small values of $t_{\mathrm{eq}}$, and thus the ions were heated more efficiently for the $m=3$ case. These results show that for lasers with the same total energy, the ions are heated better by using a slowly rising pulse shape that prevents expansion than by using a constant laser pulse which adds energy too quickly and causes the electron and ion temperatures to decouple and the plasma column to expand.

For the next part of the investigation, a $\theta$ pinch plasma heated by an intense $\mathrm{CO}_{2}$ laser beam was analyzed. The plasma was again assumed to be deuterium with an initial density of $5 \times 10^{17} \mathrm{~cm}^{3}$, initial diameter of $1.0 \mathrm{~cm}$, and initial $T_{e}$ and $T_{i}$ of $10 \mathrm{eV}$. The current was a ramp function with a maximum of $5, \times 10^{6}$ A occurring at 0.3 $\mu \mathrm{sec}$. From the pulse shape analysis discussed previously, we had concluded that a slowly rising laser pulse was the most desirable in heating a $\theta$ pinch, provided the current was also a function of time. Thus, we chose the $\mathrm{CO}_{2}$ laser light to be a quadratic $(m=2)$ shape with its peak occurring at $0.7 \mu \mathrm{sec}$. The peak laser power was $7.85 \times 10^{11} \mathrm{~W}$, and with a focal radius of 0.5 $\mathrm{mm}$ the peak laser intensity was $10^{14} \mathrm{~W} / \mathrm{cm}^{2}$. The du- ration of the analy sis was $0.9 \mu \mathrm{sec}$, which resulted in a total laser energy of $340 \mathrm{~kJ}$ being delivered to the plasma. The length of the $\theta$ pinch plasma column was $20 \mathrm{~cm}$.

In Fig. 5 the average electron and ion temperatures vs time are shown for this $\mathrm{CO}_{2}$ laser heated $\theta$ pinch. Both $T_{e}$ and $T_{i}$ are slowly rising functions for the duration of the analysis, with $T_{e}$ reaching $2 \mathrm{keV}$ and $T_{i}$ reaching slightly above $400 \mathrm{eV}$. The temperatures were found to oscillate somewhat as the plasma column pinched and expanded, but they did not seem to have reached a steady state by the end of the run, as was found for the $z$-pinch case of Fig. 1. At early times the temperatures were very close due to the small values of the equilibration time. However, by about $0.2 \mu \mathrm{sec}$ they had started to decouple as the increasing laser intensity deposited more energy into the electrons. It was found that the laser energy was deposited fairly uniformly throughout the heating time, in contrast to the $z$ pinch case where the laser energy was deposited very quickly in the plasma. The results of the heating shown in Fig. 5 indicate that the temperatures would continue to rise if the plasma were heated further, although it might be more reasonable to stop the laser after about $1 \mu \mathrm{sec}$ and then further heat the plasma by compression, as we proposed for the $z$-pinch case. Hence, it appears that a dense $\theta$

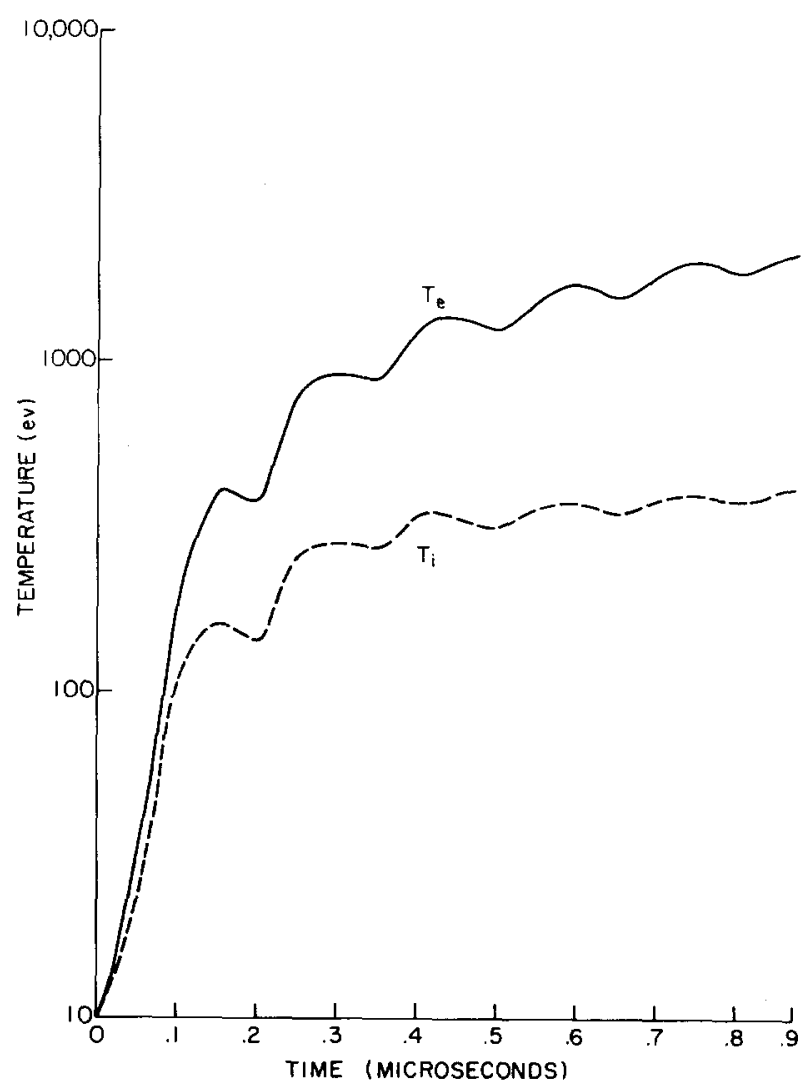

FIG. 5. Theta pinch temperatures for intense $\mathrm{CO}_{2}$ laser. 


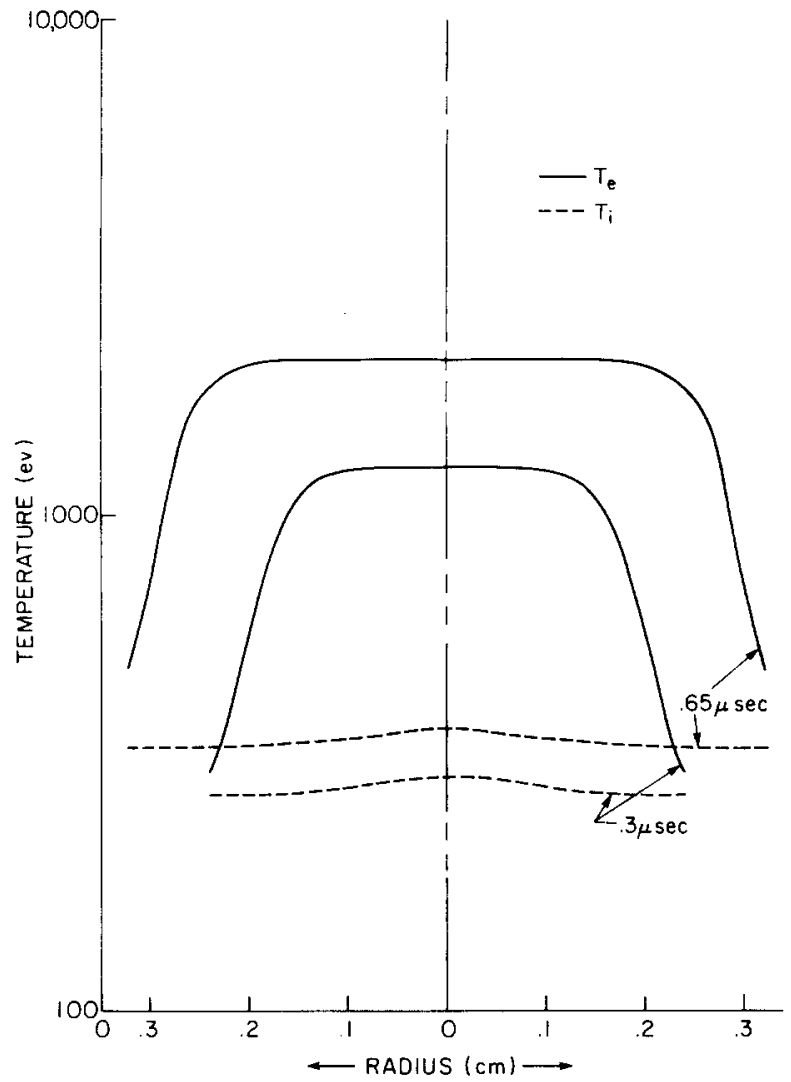

11G. 6. Theta pinch temperature profiles for intense $\mathrm{CO}_{2}$ laser.

pinch can be efficiently heated by an intense $\mathrm{CO}_{2}$ laser.

The radial distributions of the electron and ion temperatures at two different times for the $\theta$ pinch are shown in Fig. 6. It is evident that $T_{e}$ is constant across the middle of the plasma but decreases toward the boundary. In contrast, $T_{i}$ is found to be nearly constant across the plasma, although at a lower temperature than $T_{e}$. The reason that $T_{e}$ is not uniform across the plasma is the large value of the magnetic field, particularly on the outer part of the plasma. Fields of about $200 \mathrm{kG}$ occur near the edge of the plasma, while at the center the field is only a few hundred Gauss. These large fields at the outside cause a decrease in the thermal conductivity, ${ }^{13}$ so that the thermal diffusion process is not fast enough to spread the energy across the entire plasma. Since $T_{p}$ was lower on the outer region, the equilibration time was also lower. Thus, there is a more efficient transfer of energy from the electrons to the ions at that region of the plasma, and the ion temperature profiles are fairly uniform. The calculated radial density profiles were again found to be of a favorable shape, ${ }^{15}$ that is, with a minimum on the axis, for this laser heated $\theta$ pinch. The density was usually in the range of $10^{18}$ $\mathrm{cm}^{-3}$ because the plasma was compressed due to the rising current and laser pulse.

For these densities, plus the intense $\left(10^{14} \mathrm{~W} / \mathrm{cm}^{2}\right)$ laser beam, it may be possible to excite some type of anomalous heating instability mechanism in the plasma. To simulate the effects of anomalous heating in this $\theta$ pinch plasma, we used the temperature cutoff $\left(T_{c}\right)$ model in which the classical inverse bremsstrahlung absorption is increased only when $T_{e}>T_{c}$. We studied the effect on the average electron and ion temperatures in comparison to the classical heating for $T_{c}=100$ and $500 \mathrm{eV}$. It was found $^{23}$ that $T_{e}$ is about the same for early times, as expected since the absorption is increased only when $T_{e}$ exceeds $T_{c}$. However, once $T_{e}$ reaches the cutoff value there is a very great increase in $T_{e}$ for both cases, as compared with the classical. This behavior is in contrast to the anomalous heating results for the $z$ pinch case, where the absorption was increased quickly using the scaling method. Because of the slowly rising laser pulse, by the time the anomalous absorption had an ef fect, the plasma was able to be contained, and most of the increased absorption went into heating of the electrons instead of into motion of the plasma. We found that there was not very much difference in the ion temperature for the cases because of the large value of the equilibration time. Thus, the energy transfer was poor and the very hot electrons were not able to transfer much of their energy to the ions.

\section{SUMMARY AND CONCLUSIONS}

We have developed a magnetohydrodynamic model to describe the dynamics of both linear $z$-pinch and $\theta$-pinch plasmas as they are axially heated by a laser beam. The system of equations in one-dimensional (radial), cylindrical geometry were then solved numerically by a computer code. The results of the simulation of using $\mathrm{CO}_{2}$ lasers to heat dense pinches indicate that the two most important parameters in obtaining efficient heating are the temporal shapes of the laser pulse and the electric current. By careful tailoring of these quantities, efficient heating of dense pinch plasmas by $\mathrm{CO}_{2}$ lasers was possible. If these quantities are not programmed carefully, the electrons may heat very quickly, in which case the electron-ion equilibration time increases, and there is poor transfer of energy from the electrons to the ions. Also, if the heating is too fast, the plasma column may just expand with little of the laser energy being used to raise the plasma temperature. This possible decoupling of the electron and ion temperatures is an important consideration in obtaining efficient heating of pinches by intense lasers.

The pinches simulated in this research were short $(20-50 \mathrm{~cm})$ because the model was only one dimensional. Thus, the analysis pertained more to a small scale experiment than a possible reactor configuration, although these simulations could also be regarded as applying to a short section of a long reactor. The calculations were made for a short laser pulse, while a reactor would require much longer confinement times. However, it was found that the laser light absorption was most important during the early stages of confinement, when the temperatures are lowest. The most viable scheme seems to be one in which the laser is used only in this early stage of heating, before the absorption becomes too poor, thus easing the requirements on the laser. The plasma could then be further heated by compression during the later stages of confinement. During these later times one can also expect much better energy trans- 
fer between the electrons and ions, and plasma temperatures in the range of 5 to $10 \mathrm{keV}$ should be possible.

\section{ACKNOWLEDGMENT}

This work was supported in part by the National Science Foundation.

* Present address: Plasma Physics Laboratory, Princeton University, Princeton, N. J. 08540.

${ }^{1} \mathrm{~J}$. Nuckolls, L. Wood, A. Thiessen, and G. Zimmerman, Nature 239, $139(1972)$.

${ }^{2}$ J. M. Dawson, A. Hertzberg, R. Kidder, G. C. Vlases, H. G. Ahlstrom, and L. Steinhauer, in Plasma Physics and Controlled Thermonuclear Research (International Atomic Energy Agency, Vienna, 1971), Vol. I, p. 673.

${ }^{3}$ J. M. Dawson, Bull. Am. Phys. Soc, 19, 421 (1.974).

${ }^{4}$ C. Cason, G. J. Dezenberg, and R. J. Huff, Appl. Phys, Lett. 23,110 (1973).

${ }^{5}$ M. C. Richardson, K. Leopold, and A. J. Alcock, IEEE J. Quantum Electron. QE-9, 934 (1973).

${ }^{6}$ A. S. Engelhardt, V. Fuchs, C. R. Neufeld, C. Richard, and R. Decoste, Appl. Phys. Lett. 20, 425 (1972).

${ }^{7}$ N. A. Amherd and G. C. Vlases, Bull. Am. Phys. Soc. 18, 1326 (1.973).
${ }^{8}$ G. C. Vlases, Phys. Fluids 14, 1287 (1971).

${ }^{9}$ D. R. Cohn and B. Lax, Appl. Phys. Lett. 21, 21.7 (1972).

${ }^{10}$ K. Hain, G. Hain, K. V. Roberts, S. J. Roberts, and W. Koppendorfer, Z. Naturforsch. A 15, 1039 (1960).

${ }^{11}$ K. V. Roberts and D. E. Potter, in Plasma Physics, edited by B. Alder, S. Fernbach, and M. Rotenberg (Academic, New York, 1970), p. 339.

${ }^{12}$ L. Spitzer, Jr., Physics of Fully Ionized Gases (Inter science, New York, 1962), 2nd ed., Chap. 5.

${ }^{13}$ S. J. Braginskii, in Reviews of Plasma Physics, edited by M. A. Leontovich (Consultants Bureau, New York, 1.965), Vol. 1, p. 21.7 .

${ }^{14} \mathrm{R}$. D. Richtmyer and K. W. Morton, Difference Methods for Initial Value Problems (Interscience, New York, 1967), p. 313.

${ }^{15}$ L. C. Steinhauer and H. G. Ahlstrom, Phys. Fluids 14, 1.109 (1971).

${ }^{16}$ R. E. Kidder, in Physics of High Energy Densities, edited by P. Caldirola (Academic, New York, 1971), p. 306.

${ }^{17}$ P. K. Kaw and J. M. Dawson, Phys. Fluids 12, 2586 (1969).

${ }^{18}$ E. A. Jackson, Phys. Rev, 153, 235 (1.967).

${ }^{19}$ V. P. Silin, Zh. Eksp. Teor. Fiz. 51, 1842 (1966) [Sov. Phys. -JETP 24, 1.242 (1967)].

${ }^{20}$ W. L. Kruer, Phys. Fluids 16, 1548 (1973).

${ }^{21}$ D. W. Forslund, J. M. Kindel, and E. L. Lindman, Phys. Rev. Lett. 30, 739 (1973).

${ }^{22} \mathrm{~J}$, W. Shearer and J. J. Duderstadt, Nucl. Fusion 13, 401. (1973).

${ }^{23}$ D. L. Chapin, Ph. D. thesis, University of Michigan (1.974). 